\title{
Pre-authentication for Fast handoff in Wireless mesh networks with mobile APs
}

\author{
Chanil Park, Junbeom Hur, Chanoe Kim, Young-joo Shin, and Hyunsoo Yoon \\ Korea Advanced Institute of Science and Technology, Daejeon, Korea \\ \{chanil, jbhur, cokim, yjshin, hyoon\}@nslab.kaist.ac.kr
}

\begin{abstract}
Wireless mesh networks can extend the network service region by just adding APs. However wireless mesh networks also have the same security problems as the traditional wireless LAN. Until now, many methods have been proposed to solve the authentication problem, particularly for the fast handoff, in the traditional wireless LAN. However, previous methods are not efficient to the wireless mesh network with mobile APs because they just considered static APs. In this paper, we propose a new pre-authentication method for the wireless mesh network with mobile APs. We adapted the neighbor graph method of previous schemes for the compatibility. However, our method is suitable to the wireless mesh network by applying a Du et al's key distribution. Furthermore, we present a formal analysis about our method by using a logic based formal analysis method.
\end{abstract}

\section{Introduction}

In the wireless mesh networks, access points(AP) are deployed to cover a region where wireless network services are desired. But unlike the traditional wireless LAN, APs are not directly connected to the wired networks. They are connected via wireless links to form a wireless LAN backbone. Moreover, some APs have a mobility to support dynamic services. These characteristics of connection and mobility provide a significant deployment advantage. Since by just adding APs, we can extend the wireless service region.

Open wireless network based on the IEEE 802.1X Std requires a mutual authentication when mobile users, we call them supplicant(STA), want to connect to the network via nearby AP. The mutual authentication protects an invalid user's access and enhances a secure communication between STA and APs. However, authentication process consumes more time, so that seamless services for real-time application such as VoIP are sensitive to the authentication process when they handoff over APs. Actually, authentication among the STA, Authentication Server(AS), and AP in the wireless LAN cost almost $750 \mathrm{~ms}$ in the best case and $1200 \mathrm{~ms}$ in the worst case [1].

Pre-authentication methods for the fast connection association with APs, especially when STA handoff among APs, have been studied in order to reduce the handoff latency $[13,8,9,11]$. For the pre-authentication, the AS predistributes an authentication context such as Pairwise Master Key(PMK) to the neighbor 


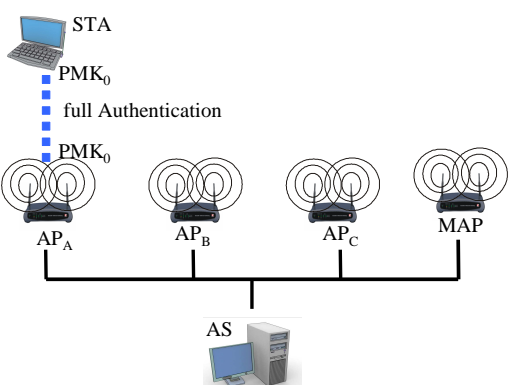

(a) Full authentication between STA and AS

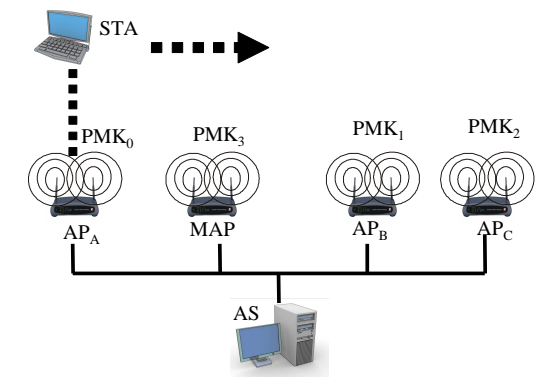

(c) MAP move between $\mathrm{AP}_{\mathrm{A}}$ and $\mathrm{AP}_{\mathrm{B}}$ and $\mathrm{STA}$ move to next access point

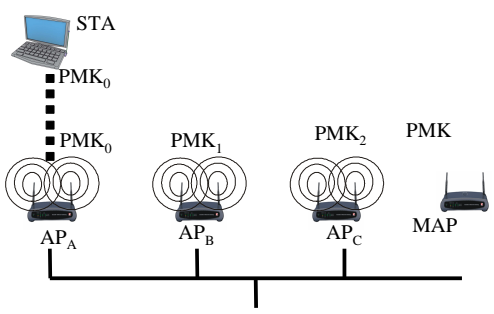

(b) Pre-distribute PMK Tree for the fast authentication

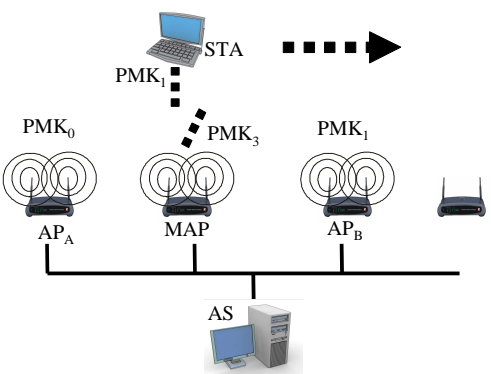

(d) Break the reauthentication

Fig. 1. The problem of pre-authentication in the mesh network consists of moving APs

APs which a STA may handoff. These PMKs should have different values per each AP and the roaming STA also should confirm that he has the same secret value with next connecting AP as he is roaming over the wireless LAN. The method using the IAPP [7] is efficient when APs are static. But mobile APs are different from APs in the IAPP [13]. First, the mobile AP does not need to maintain the neighbor graph for each AP. Second, in IAPP, multiple copies of pre-authentication context are distributed to neighbor APs, while mobile AP only forwards one copy of context block to the designated new AP. Third, STA is unaware of the mobile AP mobility management operations. Mishra et al also proposed a method for the fast handoff in the wireless LAN [9]. But their scheme just considered the fixed APs that does not move. Hence, when we apply Mishra et al's method to the wireless mesh networks which have mobile APs, we need some additional conditions. First, if an AP moves from one place to another place then the tree of PMKs in the Mishra et al's scheme has to be changed. It means that AS must change all the PMKs in the PMK tree of the neighbor graph. If AS does not change the PMKs in the PMK tree, then the STA has to be re-initiated with full authentication via AS because of breaking of PMK tree between AP and STA.

Fig.1 shows the problem of previous method in the wireless mesh network with a mobile AP. For example, (a) Let STA moving from $A P_{A}$ to $A P_{C}$ first 


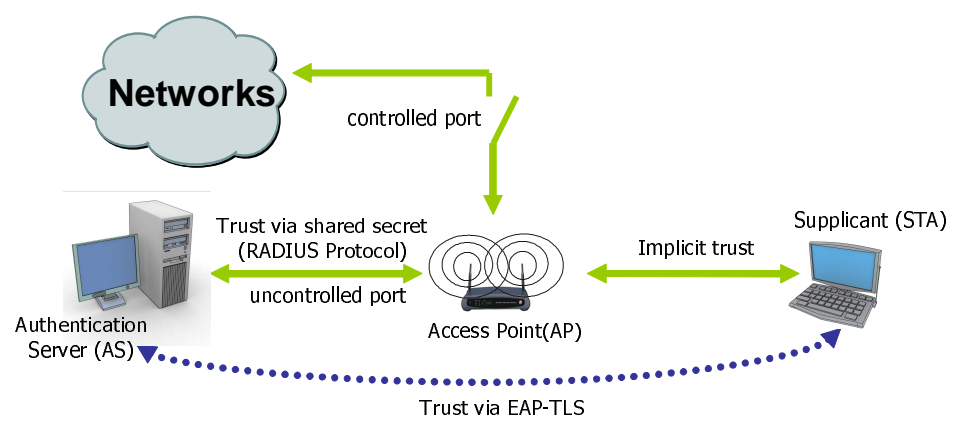

Fig. 2. IEEE $802.1 \mathrm{x}$ architecture

associated with $A P_{A}$. (b) Then AS has to predistribute the PMKs to next APs, respectively. (c) At this situation let a mobile AP, called $M A P$, move to between the $A P_{A}$ and the $A P_{B}$. (d) Then when STA moves to a next MAP, STA will make a new $P M K_{1}$ by the PMK chain. But the MAP will has a different value $P M K_{3}$ if AS would not redistribute $P M K_{1}$ to the MAP. Therefore, the STA and the MAP can not authenticate each other since the STA and the MAP do not share the authentication information. Hence STA has to re-authenticate with a full authentication via AS.

The goal of this paper is to design a pre-authentication scheme for fast handoff in the wireless mesh network. We improved a previous methods, especially Mishra et al's, to apply to the wireless mesh networks. Our scheme also makes a group of PMKs. However, PMKs in our scheme does not have a chain relation between two continuous PMKs in the neighbor graph. But roaming STAs are able to generate the same PMK with current and next connecting APs without the help of AS. For our method, we applied Du et al's key pre-distribution method [3] to make PMK and we slightly modified the pre-authentication and 4-way handshakes in the IEEE 802.11i Standard [8]. Furthermore, we present a formal analysis about our method.

The remainder of this paper is organized as follows. In Section 2, we give some related works. In Section 3, we describe the details of our protocol. In Section 4, we analyze the security of our protocol. We conclude the paper in Section 5 .

\section{Related work}

\subsection{IEEE 802.11 authentication architecture}

IEEE 802.1x provides a framework for the authentication and authorization to the wireless devices connecting to the wireless networks. IEEE 802.1x controls wireless services through the concept of controlled and uncontrolled ports at 


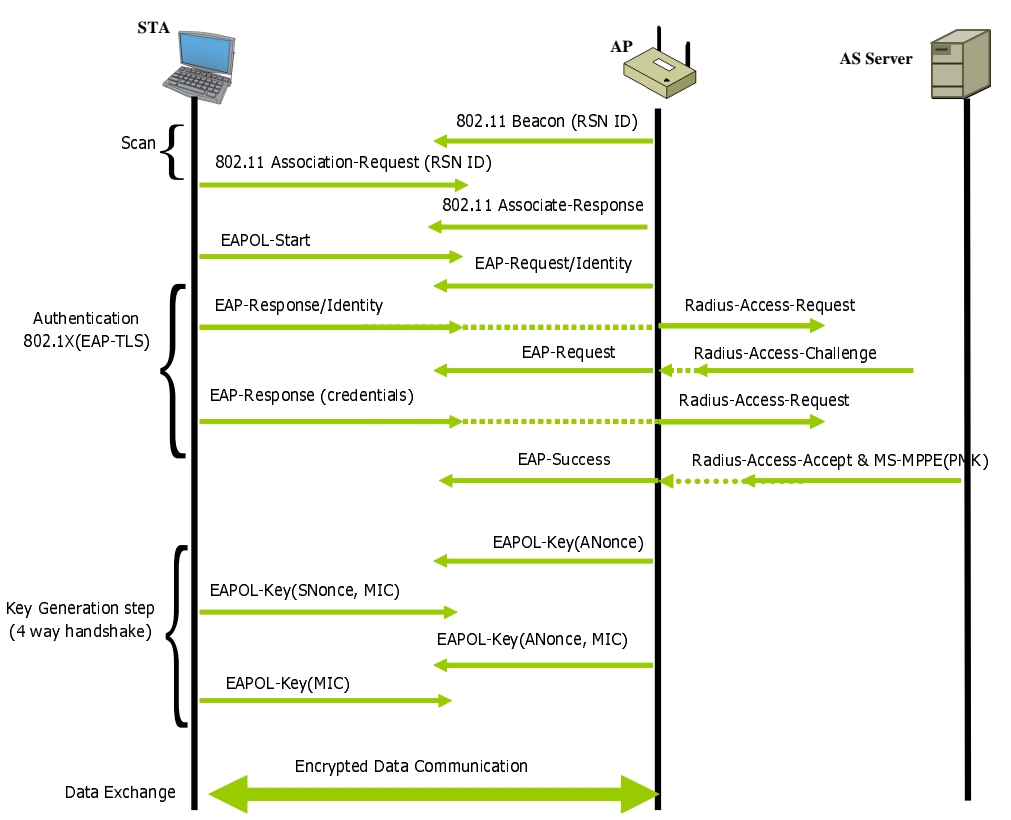

Fig. 3. IEEE 802.11i authentication flow

the layer 2 level. IEEE 802.1x consists of three main components: Supplicant, Authenticator, and Authentication server. Fig.2 shows the IEEE 802.1x architecture. The IEEE 802.11i standard [8] defines how to control the authentication for the connection among the three main components in the IEEE 802.11 networks. In the IEEE 802.11i, AP acts as a authenticator and the STA plays a role of supplicant.

The AS and AP mutually authenticates via the RADIUS protocol [12]. The uncontrolled port in the AP is used to forward the authentication data between the STA and the AS. After AS has successfully performed the mutual authentication with the STA, the AS informs an authentication information including PMK to the AP. Then, AP and STA mutually authenticate each other by EAPOL-key exchange. At this point, if the key exchange is successful, AP and STA share the same established key called pairwise temporal key(PTK) and AP allows traffics from the STA to flow through the controlled port. Fig. 3 shows the IEEE 802.11i protocol for the mutual authentication among the STA, AP, and AS. STA has to be authenticated by the AS. This protocol consists of four steps: scan, authentication, key generation and data communication. The first step is the STA's association to find suitable APs. The STA responds to the AP's beacon with an association-request message. For this message, AP also responds with the association-respond message. Since STA has to find the strongest signal among 
the neighbor APs, this step costs the most time in the wireless connection during the handoff. In the second step, STA and AS mutually authenticate via the selected AP. If this step is completed successfully, the STA and the AS can make a shared authentication context. This step is executed with the EAP protocol [2]. At the last message of this step, AS transmits the authentication information including PMK to the AP. The third step is authentication step between the STA and the AP. This step confirm that the AP is the legal device which is controlled by the AS. After these step, STA and AP share the session key.

\section{$2.2 \quad$ Fast handoff using neighbor graphs}

Recently, Mishra'et al [9] proposed pro-active key distribution using neighbor graph for the fast handoff. His work reduces the latency of the authentication phase by pre-distributing key materials ahead of a mobile subscriber's handoff. Mishra'et al's approach provides all of the same properties of a full EAP/TLS authentication which is in the IEEE 802.11i standard, but at significantly less cost in terms of latency and computational power of the mobile station.

PMK tree Mishra et al's scheme used a tree of PMKs according to a STA's roaming. In the current 802.11 f framework the PMK is derived from the shared Master Key(MK) between the STA and AS. But Mishra'et al changed the derivation of the PMK to the recurrence shown in the equation (1), where $n$ represents the $n^{\text {th }}$ reassociation for $n \geq 0$.

For example, let the placement of APs and the corresponding neighbor graph is like the Fig.4. Then the neighbor APs of $A P_{A}$ are $A P_{B}$ and $A P_{E}$, and the neighbor APs of $A P_{B}$ are $A P_{E}, A P_{C}$, and $A P_{D}$. Consider a STA who first connected at the $A P_{A}$ moves to the $A P_{D}$ through the $A P_{C}$. In this situation, the moving STA needs to handoff to the $A P_{B}$ and $A P_{C}$ successively and finally reached to the $A P_{D}$. Therefore the distributed PMKs need to be composed like the Fig.5.

$$
\begin{aligned}
P M K_{0}= & T L S-P R F(M K, " \text { clientEAPencryption" } \mid \\
& \text { clientHello.random } \mid \text { serverHello.random }) \\
P M K_{n}= & T L S-P R F\left(M K, P M K_{n-1}\left|A P_{M A C}\right|\right. \\
& \left.S T A_{M A C}\right)
\end{aligned}
$$

where $A P_{M A C}$ and $S T A_{M A C}$ are mac addresses of AP and STA, respectively. The recurrence shown in equation (1) creates a PMK tree with the reassociation pattern, $\Gamma(S T A)$, which represents a path within the tree as shown in the Fig.5. In Fig.5, the reassociation pattern is $\Gamma(S T A)=A, B, C, D$.

PMK distribution After STA and AS complete the initial full authentication via the AP connected by the STA, AS sends an ACCESS-ACCEPT message to the AP indicating successful completion of the authentication process as we 

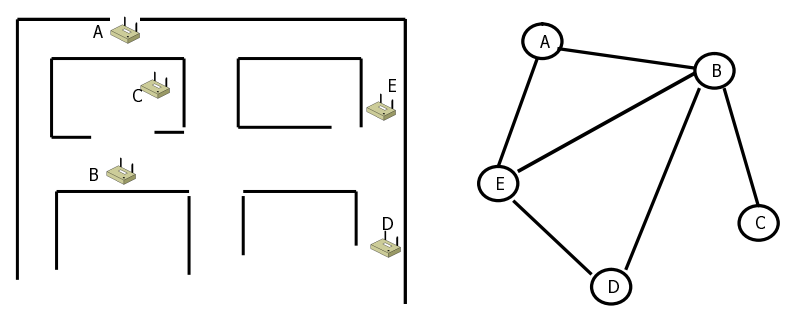

Fig. 4. Example of the placement of APs and the corresponding neighbor graph

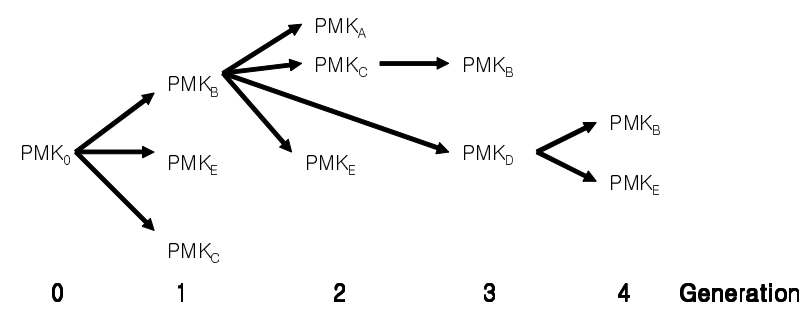

Fig. 5. PMK tree

seen in the Fig.2. At this point AS sends an $P M K_{0}$ with ACCESS-ACCEPT message. After that AS determines the neighbors of AP currently associated by the STA and sends to them a NOTIFY-REQUEST that a specific mobile station may roam into the coverage area of each of the neighboring APs. Neighbor APs may decide to request the security association. If a neighbor AP decides to request the PMK, then the neighbor AP sends a NOTIFY-ACCEPT message to the AS. If not, the AP sends a NOTIFY-REJECT message to the AS. AS responds to the neighbor APs with an ACCESS-ACCEPT message which contains the appropriate PMK as well as authorization for the user to remain connected to the network.

\subsection{Key pre-distribution scheme}

Blom proposed a key predistribution scheme that allows any two pairs in the network to derive a pairwise secret key [3]. Blom's scheme has the following special property: all communication links of non-compromised pairs remain secure along as no more than $h$ pairs are compromised, which called a $h$-secure. Du modified the scheme of Blom in order to make it suitable for sensor networks [5]. Key generation process of Du'et al is as follows: Let $M$ be a matrix of size $(h+1) \times N$ over finite field $G F(q)$, where $N$ denotes the size of the network and $q(\gg N)$ is a large prime number. Matrix $M$ is a public information shared among 

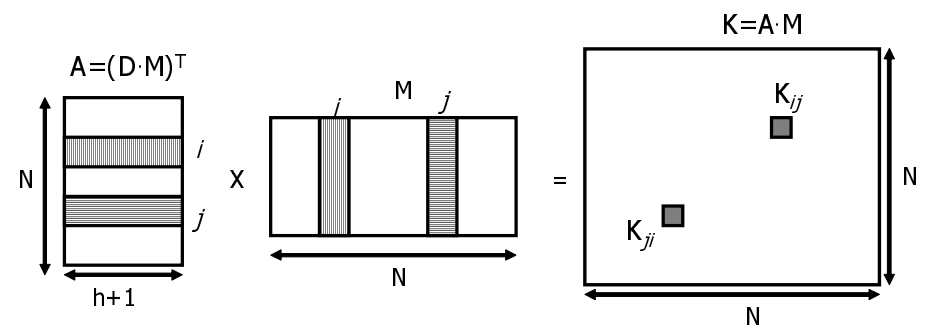

Fig. 6. Shared key generation in Blom's scheme:

the participants. In the key generation phase, Key Distribution Center(KDC) creates a random symmetric matrix $D$ of size $(h+1) \times(h+1)$ over $G F(q)$, and computes an matrix $A=(D \cdot M)^{T}$, where $(D \cdot M)^{T}$ is the transpose of $D \cdot M$. Matrix $A$ must be kept securely. If we let $K=A \cdot M$, we know that $K$ is a symmetric matrix of size $N \times N$ because of the symmetric property of D. We call $K$ a key space. Fig.6 shows how the pairwise key is generated. Participants $i$ and $j$ in the networks store $A(i)$ and $A(j)$ rows of matrix $A$, respectively. When they need a shared key, they exchange their columns $M(i)$ and $M(j)$ in the matrix $M$ and compute $K_{i j}(=A(i) \cdot M(j))$ and $K_{j i}(=A(j) \cdot M(i))$ by using their private rows $A(i)$ and $A(j)$, respectively. The keys $K_{i j}$ and $K_{j i}$ become the same value because matrix $K$ is symmetric. Since $M$ is a public information, its columns can be transmitted in plaintext.

\section{Pre-authentication using different keys}

In this section, we describe the notion of our pre-authentication applying to the wireless mesh networks. Basically, our scheme follows the IEEE 802.1X authentication framework and uses the method of neighbor graph proposed by the Mishra et al. However, we do not directly distribute PMKs to APs. Instead, the AS sends a secret data which can make a shared secret key during a 4-way handshake. It has advantages that neighbor APs can not make a shared secret key like PMK before associating with a STA and PMKs using secret value do not have any chain relation between each other. Therefore our scheme can protect PMK from leaking by mobile AP. Hence, our method is more suitable for the wireless mesh network including mobile APs.

\subsection{Key generation for the pre-authentication}

In our method, we use the Du et al's key distribution method[5]. At the system initialization, we assume that AS knows the size of network in the wireless mesh 


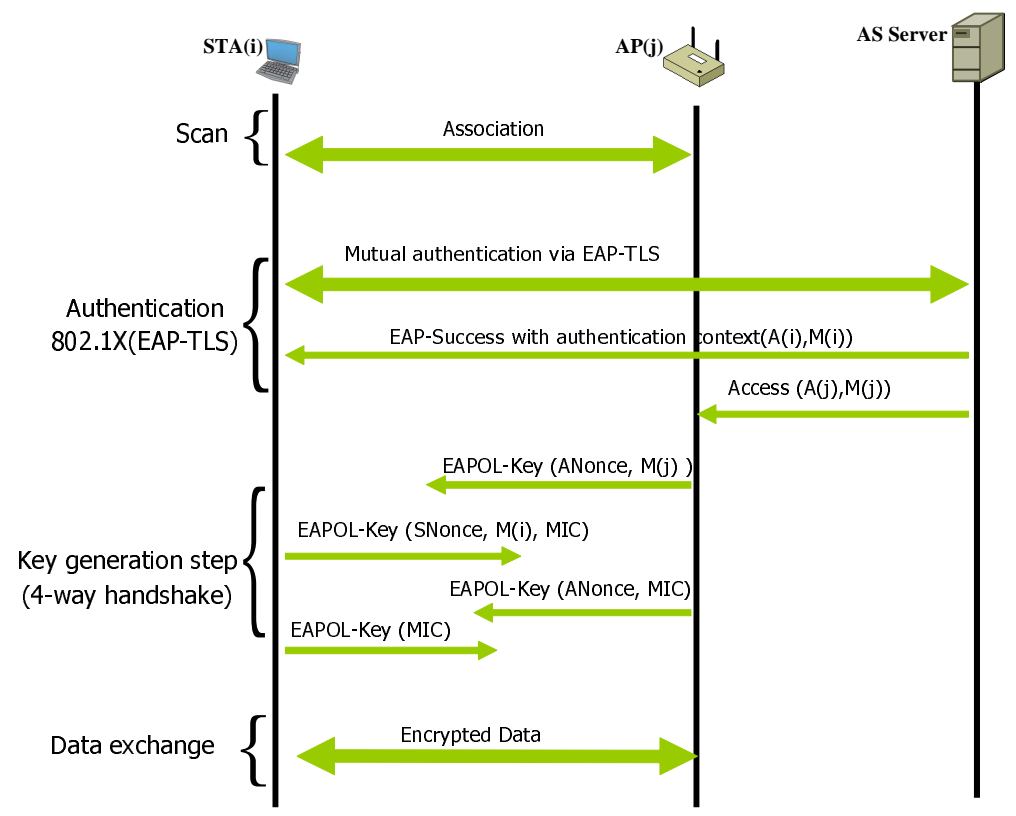

Fig. 7. Authentication flow among STA, AP, and AS in our scheme

networks which it has to control. It means that the AS knows the number of APs. So AS firstly makes a matrix $M$ size of $(h+1) \times N$ where $N$ is the number of APs controlled by the AS and $h$ is a threshold for the $h-$ secure property. Our pre-authentication is as follows (see Fig.7)

1. STA first mutually authenticates with AS to connect to the network via a AP using the IEEE 802.1X. After AS successfully completes an initial full authentication with the STA, AS generates a symmetric matrix $D$ size of $(h+1) \times(h+1)$ over finite field $G F(q)$, and computes a secret key matrix $A$ like in Sect. 2.3. The matrix $A$ becomes the key space for the STA's handoff.

2. After making the key space $A$, the AS sends to the STA a row $A(i)$ of the matrix $A$ and a column $M(i)$ of the matrix $M$, and also sends a row $A(j)$ of the matrix $A$ and a column $M(j)$ of the matrix $M$ to the current AP associating with the STA where $i$ and $j$ are the identification of STA and AP, respectively

3. At this point, the AS also sends to neighbor APs, to which the STA may handoff, of current $\mathrm{AP}$ different rows $A(k)$ of the matrix $A$ and columns $M(k)$ of the matrix $M(k \neq i, j)$, respectively.

4. After received the rows and columns, the STA and AP execute a 4-way handshake to guarantee the shared session key and to generate a temporal pairwise key. 
5. When the STA handoff to a neighbor AP, the STA and the new AP also execute a new 4-way handshake to synchronize the shared session key and temporal pairwise key.

\subsection{PMK confirmation on the wireless mesh network}

In our scheme, the 4-way handshake follows the IEEE 802.11i Standard except an additional data exchange such as columns in the matrix $M$. Our 4-way handshake is follows:

1. AP $\rightarrow$ STA: AP sends the first message. It contains a PNonce- a nonce value generated by the AP and the column $M(j)$ - the column of the matrix $M$ which is sent previously by the AS. After the STA has received this message, STA can compute the shared key $K_{i j}(=A(i) \cdot M(j))$ and temporal pairwise key, PTK, by the equation 2 . The shared key $K_{i j}$ acts a role of PMK in the IEEE 802.11i Standard

2. STA $\rightarrow$ AP: The STA sends to the AP a message containing SNonce - a nonce value generated by the AP, the column $M(i)$ of the matrix $M$, and message integrity code(MIC) over the message to protect its integrity. The AP uses the column $M(i)$ to generate a shared key $K_{j i}(=A(j) \cdot M(i))$. This shared key $K_{j i}$ is of the same value as the key $K_{i j}$ of the STA by the Blom's scheme. The AP also uses SNonce and the key $K_{j i}$ to generate the temporal pairwise key and verifies the MIC

3. AP $\rightarrow \mathrm{STA}$ : The AP sends a response message again. This message includes the earlier PNonce and MIC check which can be verified by the STA.

4. STA $\rightarrow$ AP: This message signifies the completion of the 4-way handshake and signals the installation of the keys by both entities for the data communication

The way to make a temporal pairwise key, PTK, is follows.

$$
P T K=P R F(K, P N o n c e|S N o n c e| P M A C \mid S M A C)
$$

where, PNonce and SNonce are nonces of the AP and the STA, respectively, and PMAC and SMAC are MAC address of the AP and the STA, respectively. K is the shared key between STA and AP.

\subsection{An example of matrix $M$}

We show an example of matrix $M$ that used in Du et al's work[5]. That matrix also can be applied to our scheme. In order to achieve the $h$-secure property, $h+1$ columns of $M$ must be linearly independent. Let $s$ be a primitive element of $G F(q)$; that is, each nonzero element in $G F(q)$ can be represented by some 
power of $s$. A feasible $M$ can be designed as follows [10]

$$
M=\left(\begin{array}{ccccc}
1 & 1 & 1 & \cdots & 1 \\
s & s^{2} & s^{3} & \ldots & s^{N} \\
s^{2} & \left(s^{2}\right)^{2} & \left(s^{3}\right)^{2} & \cdots & \left(s^{N}\right)^{2} \\
\vdots & \vdots & \vdots & \ddots & \vdots \\
s^{\lambda} & \left(s^{2}\right)^{\lambda} & \left(s^{3}\right)^{\lambda} & \cdots & \left(s^{N}\right)^{\lambda}
\end{array}\right)
$$

Since $M$ is a Vandermonde matrix, it can be shown that any $h+1$ columns of $M$ are linearly independent. This matrix $M$ has the nice property that its columns can be generated by an appropriate power of the primitive elements $s$. This is, to store the $k^{\text {th }}$ column of $M$ at $A P_{k}$ or STA we need only store the seed $s^{k}$ at the device which can regenerate the column.

\section{Analysis of proposed protocol}

Basically, our method follows the Mishra et al's method. However, we broke the chain relation between PMKs in the neighbor APs. So, our method is efficient in the wireless mesh network with mobile APs in spite of adapting the neighbor graph in the Mishra's method. Here, we analyze the computation overhead and memory usage. Furthermore, we presents a formal security analysis by employing the logic-based formal analysis approach $[4,6]$.

\subsection{Computational overhead and memory usage}

In our model, $\operatorname{STA}(i)$ and $\operatorname{AP}(j)$ have to calculate a shared common key $K(i . e$, $K_{i j}$ or $\left.K_{j i}\right)$ per every handoff of the STA $(i)$. To calculate the shared common key, STA(or AP) needs a row $A(i)$ (or $A(j))$ of matrix $A$ and column $M(j)$ (or $M(i)$ ) of matrix $M$, respectively. If we use Vandermonde matrix $M$ as we said in Sect 3.3. the dominating computational cost for the STA and AP is $2 h-1$ multiplications where $h-1$ comes from the need to regenerate the corresponding column of matrix $M$ from a seed and other $h$ multiplications come from the product of $A(i) \cdot M(j)$ (or $A(j) \cdot M(i))$, respectively . Furthermore, this computation can be reduced to only $h$ multiplications by using the Horner's rule for polynomial evaluation.

For the memory usage, the STA and APs need to carry $h+1$ field elements for an association. Hence, the total memory usage for the STA is $h+1$, but APs are $k \cdot(h+1)$ where $k$ is a sum of STAs connecting to the current AP and STAs connecting to the neighbor APs (we do not count the seed needed to generate $M(i)$ since this can be served as the identification of the STA or AP)

\subsection{Formal analysis for proposed scheme}

Here, we show the formal analysis for the 4-way handshake in our scheme. We use logic based formal analysis method. For the description, we let STA as $S$ 
and $\mathrm{AP}$ as $P$. The initial assumptions of the proposed solution are given as follows:(see Appendix A for detail notations)

$(A 1) S \ni N_{s},(A 2) S \models \sharp N_{s},(A 2) S \ni M(i),(A 3) S \ni A(i)$,

$(A 4) S \ni P M A C,(A 5) S \ni S M A C,(A 6) S \ni P R F()$

$(B 1) P \ni N_{p},(B 2) P \models \sharp N_{p},(B 2) P \ni M(j),(B 3) P \ni A(j)$,

(B4)P $P$ PMAC, (B5)P $\ni S M A C,(B 6) P \ni P R F()$

where $N_{s}$ and $N_{p}$ denote nonces of STA and AP, respectively. $A(i)$ and $A(j)$ are rows of matrix $A, M(i)$ and $M(j)$ denote columns of matrix $M$. SMAC and PMAC denote MAC address of STA and AP, respectively. $\mathrm{PRF}()$ is a hash function generating pseudo random values.

Assumption A1 and A2 presume that $\mathrm{S}$ believes $N_{s}$ is fresh and $\mathrm{S}$ possess $N_{s}$. Assumption $\mathrm{B} 1$ and $\mathrm{B} 2$ assume that $\mathrm{P}$ also believes $N_{p}$ is fresh and $\mathrm{P}$ possess $N_{p}$. Assumption $\mathrm{A} 3$ and $\mathrm{B} 3$ implies that $\mathrm{S}$ and $\mathrm{P}$ possess secret values to make a pairwise master key $(\mathrm{PMK})$, respectively. Assumption A4 and A5 denote that $\mathrm{S}$ knows his MAC address and MAC address of AP. Assumption B4 and B5 also denote that $\mathrm{P}$ knows his MAC address and MAC address of STA.

From the message 1 in our 4-way handshake and assumption A3, we derive the following belief by applying rules P1 and P2 (see Appendix B)

$$
\begin{gathered}
\frac{S \triangleleft\left\{N_{p}, M(j)\right\}}{S \ni\left\{N_{p}, M(j)\right\}} \\
\frac{S \ni A(i), S \ni M(j)}{S \ni K=F(A(i), M(j))}
\end{gathered}
$$

where $F$ is a matrix multiplication function.

Applying rules F1 and P2, we obtain,

$$
\begin{gathered}
\frac{S \models \sharp N_{s}}{S \models \sharp\left(N_{s}, N_{p}\right)} \quad(F 1) \\
\frac{S \ni N_{s}, S \ni N_{p}, S \ni P M A C, S \ni S M A C, S \ni K, S \ni P R F()}{S \ni P T K=P R F\left(K, N_{p}, N_{s}, P M A C, S M A C\right)} \\
\frac{S \models \sharp\left(N_{s}, N_{p}\right)}{S \models \sharp P T K} \quad(F 1) .
\end{gathered}
$$

From the message 3 and results of equation 7 and 8, we can obtain following states by invoking P1, F1, and I1

$$
\begin{gathered}
\frac{S \triangleleft\left\{N_{p}, M I C\right\}}{S \ni\left\{N_{p}, M I C\right\}} \\
S \models \sharp(P T K) \\
S \models \sharp M I C=H\left(P T K, N_{p}\right)
\end{gathered}
$$


where $H$ is a hash function

$$
\begin{gathered}
S \triangleleft * M I C, S \ni P T K, S \models S \longleftrightarrow{ }_{P T K} P, \\
S \models \phi(M I C), S \models \sharp(M I C, P T K)
\end{gathered}
$$

Hence, STA can successfully infer that the AP has the right shared PTK.

In the same way, AP can get the corresponding belief from the following states. From the message 2 in our 4-way handshake and assumption B3,

$$
\begin{gathered}
\frac{P \triangleleft\left\{N_{s}, M(i), M I C\right\}}{P \ni\left\{N_{s}, M(i), M I C\right\}} \\
P \ni A(j), P \ni M(i) \\
P \ni K=F(A(j), M(i))
\end{gathered}
$$

where $\mathrm{F}$ is a matrix multiplication function.

Applying rules F1 and P2, we obtain,

$$
\begin{gathered}
\frac{P \models \sharp N_{p}}{P \models \sharp\left(N_{s}, N_{p}\right)} \quad(F 1) \\
\frac{P \ni N_{s}, P \ni N_{p}, P \ni P M A C, P \ni S M A C, P \ni K, P \ni P R F()}{P \ni P T K=P R F\left(K, N_{p}, N_{s}, P M A C, S M A C\right)} \\
\frac{P \models \sharp\left(N_{s}, N_{p}\right)}{P \models \sharp P T K} \quad(F 1) .
\end{gathered}
$$

From results of equation 15 and 16, we can obtain following states by invoking F1 and I1

$$
\frac{P \models \sharp(P T K)}{P \models \sharp M I C=H\left(P T K, N_{s}\right)}
$$

where $H$ is a hash function

$$
\begin{gathered}
P \triangleleft * M I C, P \ni P T K, P \models P \longleftrightarrow P T K S, \\
P \models \phi(M I C), P \models \sharp(M I C, P T K)
\end{gathered}
$$

Hence, AP can also successfully infer that the STA has the right shared PTK.

\section{Conclusion}

In the paper, we proposed the pre-authentication method for fast handoff in the wireless mesh network. We broke the chain relation between PMKs in the neighbor APs in the previous methods. It makes the authentication between the 
roaming STA and mobile APs freely. Hence, our method is efficient in the wireless mesh network with mobile APs. Furthermore, we analyzed the computation overhead and memory usage. We presented the formal analysis about our method by using the logic based formal analysis.

\section{Acknowledgment}

This work was supported by the Ministry of Science and Technology(MOST) /Korea Science and Engineering Foundation(KOSEF) through the Advanced Information Technology Research Center(AITrc) and the MIC(Ministry of Information and Communication), Korea, under the ITRC(Information Technology Research Center) support program supervised by the IITA(Institute of Information Technology Assessment)

\section{References}

1. Alimian, A. and Aboba, B.: IEEE 802.11-04/377r1. March (2004).

2. Aboba, B., Blunk, L., Carlson, J., and Levkowetz, H.: Extensible Authentication Protocol (EAP). RFC 3748, June (2004).

3. Blom, R.: An optimal class of symmetric key generation systems. in Proceedings of EUROCRYPT. (1984)

4. Burrows, M., Abadi, M., and Needham, R.: A logic of authentication. ACM Transactions on Computer Systems, pp.18-36, (1990).

5. Du, W., Deng, J., Han, Y., Varshney, P., Kate, J., and khalili, A.: A Pairwise Key Pre-Distribution Scheme for Wireless Sensor Networks. In Proceedings of 10th ACM Conference on Computer and Communications Security(CCS 03). pp.42-51. (2003)

6. Gong, L., Needham, R., and Yahalom, R.: Reasoning about belief in cryptographic protocols. Proceedings of the 1990 IEEE Symposium on Security and Privacy. IEEE Computer Society, Silver Spring, MD, pp.234-248, (1990).

7. IEEE 802.11F: IEEE Trial-Use Recommended Practice for Multi-Vender Access Point Interoperability via an Inter-Access Point Protocol Across Distribution System Supporting IEEE 802.11 Operation. July (2003).

8. IEEE 802.11i: Amendment 6: Medium Access Control (MAC) Security Enhancements, IEEE Computer Society, July (2004).

9. Mishra, A. Shin, M., and Arbaugh, W.: Pro-active Key Distribution using Neighbor Graphs. IEEE Wireless Communication, vol. 11, February (2004).

10. MacWilliams, F. and Sloane, N.: The Theory of Error-Correcting Codes. Elsevier Science Publishing Company, Inc.(1977).

11. Pack, S., and Choi, Y.: Fast Inter-AP Handoff using predictive authentication scheme in a public wireless LAN. In Proceedings of IEEE Networks Conference. Atlanta, GA, Aug. (2002).

12. Rigney, C.,Willens, S.,Rubens, A.,Simpson, W.: Remote Authentication Dial In User Service (RADIUS). RFC 2865, June (2000).

13. Wang, J., Bao, L.: Mobile Context Handoff in Distributed IEEE 802.11 Systems. International Conference on Wireless Networks, Communications, and Mobile Computing(WIRELESSCOM), Maui, HI, June 13-16,(2005). 


\section{Appendix A: Notation}

Here, we describe some notations used in the paper. Refer to $[4,6]$ for a detail description

\section{$7.1 \quad$ Formulae}

A formula is a name used to refer to a bit string, which would have a particular value in a run. Let $\mathrm{X}$ and $\mathrm{Y}$ range over formulae and $\mathrm{K}$ is a key

- $(X, Y)$ : conjunction of two formulae. We treat conjunctions as sets with properties such as associativity and commutativity.

- $X_{K}$ and $X_{K}^{-1}$ : conventional encryption and decryption.

$-X_{+K}$ and $X_{-K}$ : public-key encryption and decryption.

$-F\left(X_{1}, \cdots, X_{n}\right): \mathrm{F}$ is a many to one computationally feasible function.

$-H(X)$ : one-way function of $\mathrm{X}$. It is required that given $\mathrm{X}$ it is computationally feasible to compute $H(X)$; given $H(X)$ it is infeasible to compute $X$.

\subsection{Statements}

We describe some basic statements which reflects some property of a formula.

Let $\mathrm{P}$ and $\mathrm{Q}$ range over principals. The following are the basic statements

$-P \models C: \mathrm{P}$ believes that statement $\mathrm{C}$ holds.

- $P \triangleleft X$ : $\mathrm{P}$ sees $\mathrm{X}$ or $\mathrm{P}$ receives $\mathrm{X}$, possibly after performing some computation such as decryption.

$-P \ni X: \mathrm{P}$ possess $\mathrm{X}$. At a particular stage of a run, this includes all the formulae that $\mathrm{P}$ has been told, all the formulae he started the session with, and all the ones he has generated in that run.

$-P \triangleleft * X$ : $\mathrm{P}$ sees $\mathrm{X}$ or $\mathrm{P}$ receives $\mathrm{X}$ and $\mathrm{P}$ never said $\mathrm{X}$.

$-\sharp(X)$ : The formula $\mathrm{X}$ is fresh. That is, $\mathrm{X}$ has not been used for the same purpose at any time before the current run of the protocol.

$-P \models \phi(X): \mathrm{P}$ believes that formula $\mathrm{X}$ is recognizable. That is, $\mathrm{P}$ would recognize $\mathrm{X}$ if $\mathrm{P}$ has certain expectations about the contents of $\mathrm{X}$ before actually receiving $\mathrm{X}$.

$-P \mid \sim X$ : Ponce conveyed formula $\mathrm{X}$. X can be a message itself or some content computable from such a message.

$-C_{1}, C_{2}$ : Conjunction.

\section{Appendix B: Logical Postulates}

In this section we introduce the logical postulates underlying the reasoning process. There are five categories of postulates. We describe some of each category and present representative postulates. For the complete list of all postulates, refer to $[4,6]$. 


\section{Being-Told Rules}

- (T1) $\frac{P \triangleleft(X, Y)}{P \triangleleft X} \quad$ Being told a formula implies being told each of its concatenated components

- (T2) $\frac{P \triangleleft\{X\}_{K}, P \ni K}{P \triangleleft X} \quad$ If a principal is told a formula encrypted with a key he possesses then he is also considered to have been told the decrypted contents of that formula.

\section{Possessing rules}

- (P1) $\frac{P \triangleleft X}{P \ni X} \quad$ If $\mathrm{P}$ sees $\mathrm{X}$ or $\mathrm{P}$ receives $\mathrm{X}, \mathrm{P}$ possesses $\mathrm{X}$.

- (P2) $\frac{P \ni X, P \ni Y, P \ni F()}{P \ni(X, Y), P \ni F(X, Y)} \quad$ If $\mathrm{P}$ possesses each item in a group, then $\mathrm{P}$ possesses the group and the function of the group.

\section{The freshness rules}

- (F1) $\frac{P \models \sharp(X)}{P \models \sharp(X, Y), P \models \sharp(F(X))} \quad \mathrm{P}$ believes a formal $\mathrm{X}$ is fresh, then he is believe that any formula of which $\mathrm{X}$ is a component is fresh, and a computationally feasible one-to-one function $\mathrm{F}$ of $\mathrm{X}$ is fresh.

- (F2) $\frac{P \models \sharp(X), P \ni K}{P \models \sharp\left(\{X\}_{K}\right), P \models \sharp\left(\{X\}_{K}^{-1}\right)} \quad$ If $\mathrm{P}$ believes a formula $\mathrm{X}$ is fresh and possesses a key, then $\mathrm{P}$ believe that the encryption, as well as the decryption, of $\mathrm{X}$ with that key is fresh.

\section{Recognizability Rules}

- (R1) $\frac{P \models \phi(X)}{P \models \phi(X, Y), P \models \phi(F(X))} \quad$ If $\mathrm{P}$ believes a formula $\mathrm{X}$ is recognizable, then he is believe that any formula of which $\mathrm{X}$ is a component is recognizable, and a computationally feasible function $\mathrm{F}$ of $\mathrm{X}$ is recognizable.

- (R2) $\frac{P \ni H(X)}{P \models \phi(X)} \quad$ If $\mathrm{P}$ possesses formula $H(X)$ then he believes that $\mathrm{X}$ is recognizable.

\section{Message Interpretation Rules}

- (I1) $\frac{P \triangleleft *\{X\}_{K}, P \ni K, P \models P \longleftrightarrow{ }_{K} Q, P \models \phi(X), P \models \sharp(X, K)}{P \models Q|\sim X, P \models Q| \sim\{X\}_{K}, P \models Q \ni K} \quad$ If for a principal $\mathrm{P}$, all of the following conditions hold: (1) $\mathrm{P}$ receives a formula consisting of $\mathrm{X}$ encrypted with $\mathrm{K}$ and marked with a not-originated -here mark; (2) P possesses key $\mathrm{K}$; (3) P believes $\mathrm{K}$ is a secret key between him and Q; (4) P believes formula $\mathrm{X}$ is recognizable; (5) $\mathrm{P}$ believes that $\mathrm{K}$ or $\mathrm{X}$ are fresh. Then $\mathrm{P}$ believe the following: $\mathrm{Q}$ once conveyed $\mathrm{X}$; $\mathrm{Q}$ once conveyed the formula $\mathrm{X}$ encrypted with $\mathrm{K}$ and $\mathrm{Q}$ possesses $\mathrm{K}$ 